\title{
AXISYMMETRIC FINITE ANTI-PLANE SHEAR OF COMPRESSIBLE NONLINEARLY ELASTIC CIRCULAR TUBES
}

\author{
BY \\ DEBRA A. POLIGNONE AND CORNELIUS O. HORGAN \\ University of Virginia, Charlottesville, Virginia \\ Dedicated to J. K. Knowles on the occasion of his 60 th birthday
}

\begin{abstract}
The axial shear problem for a hollow circular cylinder, composed of homogeneous isotropic compressible nonlinearly elastic material, is described. The inner surf ace of the tube is bonded to a rigid cylinder while the outer surface is subjected to a uniformly distributed axial shear traction and the radial traction is zero. For an arbitrary compressible material, the cylinder will undergo both a radial and axial deformation. These axisymmetric fields are governed by a coupled pair of nonlinear ordinary differential equations, one of which is second-order and the other first-order. The class of materials for which axisymmetric anti-plane shear (i.e., a deformation with zero radial displacement) is possible is described. The corresponding axial displacement and stresses are determined explicitly. Specific material models are used to illustrate the results.
\end{abstract}

1. Introduction. Finite anti-plane shear deformations of nonlinearly elastic solids have been the subject of considerable attention. In anti-plane shear of cylindrical domains of arbitrary cross section, the displacement vector is parallel to the generators of the cylinder and is independent of the coordinates parallel to the generators. Early work on such deformations (also called generalized shear and longitudinal shear) for isotropic incompressible elastic materials is that of Adkins [1] (see also Green and Adkins [2] and Green and Zerna [3]). More recently, anti-plane shear deformations have been of interest in the context of fracture mechanics (see e.g. [4] and the references cited therein). As discussed in [4], apart from their intrinsic interest, crack problems in anti-plane shear (mode III) provide valuable guidance for investigation of the more complicated plane problems (modes I, II). Stress concentration problems in finite anti-plane shear have also been investigated [5-7].

A precise characterization of anti-plane shear deformations within the framework of three-dimensional finite elastostatics has been given by Knowles for both incompressible [8] and compressible [9] isotropic materials. As pointed out in [8,9], not all nonlinearly elastic materials can sustain finite anti-plane shear deformations in a cylinder of arbitrary cross section. Necessary and sufficient conditions on the strain-

Received October 30, 1990.

(C)1992 Brown University 
energy density $W$ for such deformations to be possible are established in [8,9]. For incompressible materials, a single condition on $W$ is obtained in [8] while for compressible materials two conditions are required [9]. Examples of particular strainenergy densities for which finite anti-plane shear is possible are provided in [8,9]. These include the neo-Hookean and Mooney-Rivlin materials in the incompressible case [8]. Since the conditions on $W$ in the compressible case are more restrictive, examples of particular admissible material models are not as readily obtained. They do include special Hadamard materials, although not the Blatz-Ko material [9].

The purpose of the present paper is to further examine this issue for compressible materials. We restrict the domain geometry to be that of a circular cylinder and confine attention to axisymmetric finite anti-plane shear deformations. In this way we are able to describe a wide variety of compressible materials for which such a restricted class of anti-plane shear deformations are possible. It should be noted that for incompressible materials, the situation is quite different and has been completely resolved. For a circular (hollow or solid) cylinder composed of an arbitrary isotropic incompressible elastic material, an exact solution to the axial shear problem giving rise to axisymmetric anti-plane shear deformations has been obtained by Adkins [1] (see also [3, Sec. 3.8; 10, pp. 296-303; 8, footnote 2, p. 409]). Denoting the radial components of a cylindrical polar coordinate system in the undeformed and deformed configurations by $R$ and $r$ respectively, the solutions described in [1, 3, $10]$ are such that

$$
r=R .
$$

The deformed configuration is again a circular cylinder. Such deformations have also been called telescopic shear $[11,12]$.

For compressible materials, deformations of the form (1.1) will not be possible for all materials. In the next section, we provide a formulation of the axial shear problem for a hollow circular cylinder, composed of an isotropic compressible elastic material, with inner surface bonded to a rigid cylinder. The outer surface is subjected to a uniformly distributed axial shear traction and the radial traction is zero. For an arbitrary compressible material, the cylinder will undergo both a radial and axial deformation. The radial deformation $r(R)$ and the out-of-plane displacement $w(R)$ are shown to be governed by a coupled pair of nonlinear ordinary differential equations, one of which is second-order and the other first-order (see equations (2.26), (2.24) below). In Sec. 3, we establish necessary conditions on the strain-energy density $W$ for axisymmetric finite anti-plane shear to be possible by seeking solutions of the governing equations for which $r=R$ so that (1.1) holds. We obtain two conditions (see (3.3), (3.4)) on $W$ in the form of a second-order and first-order nonlinear ordinary differential equation for $w(R)$ whose solutions must be compatible. In Sec. 4 a subclass of materials for which these conditions are satisfied is identified. For this subclass, the conditions reduce to algebraic restrictions on constitutive functions appearing in the representation for $W$. The out-of-plane displacement $w(R)$ is found explicitly in terms of a logarithmic function. The corresponding stresses are also obtained explicitly. Similar results have been obtained in a different fashion and for other boundary conditions in [11-13]. The subclass of materials discussed in 
Sec. 4 contains the special Hadamard materials found in [9] for which general (not necessarily axisymmetric) anti-plane shear deformations are possible in a cylinder of arbitrary cross section. The strain-energy density $W$ for these materials does not depend on the second invariant of the Cauchy-Green deformation tensor. Particular examples of these special Hadamard materials that have been proposed in the literature are described briefly in Sec. 4. It is also shown that materials for which $W$ does depend on the second invariant are admissible. In Sec. 5 a wider range of materials is examined. By broadening the subclass of materials considered in Sec. 4, we find a wide variety of strain-energies (which also depend on the second invariant) for which axisymmetric finite anti-plane shear deformations of the tube are possible.

Issues similar to those addressed here have been examined recently by the present authors [14] for finite torsional deformations of compressible nonlinearly elastic solid circular cylinders subjected to end torques. Thus the class of compressible materials for which pure torsion (i.e., a deformation with zero radial displacement) is possible is identified in [14]. The results obtained in [14] and in the present paper complement one another in the sense that many of the materials described in [14] for which pure torsion is possible do not sustain axisymmetric finite anti-plane shear while, conversely, several of the admissible materials identified here do not sustain pure torsion.

2. Formulation of the axial shear problem. We are concerned with axisymmetric finite axial shear deformation of an isotropic compressible nonlinearly elastic hollow circular cylinder. Thus the deformation, which takes the point with cylindrical polar coordinates $(R, \Theta, Z)$ in the undeformed region to the point $(r, \theta, z)$ in the deformed region, has the form

$$
r=r(R), \quad \theta=\Theta, \quad z=Z+w(R)
$$

with $\dot{r} \equiv d r / d R>0$, where $w$ is the out-of-plane displacement. Since the deformation is assumed to be axisymmetric, we have $w=w(R)$. As regards boundary conditions, we suppose that the inner surface $R=A$ is bonded to a rigid cylinder so that

$$
r(A)=A, \quad w(A)=0 .
$$

The deformation may be achieved either by prescribing $r(R)$ and $w(R)$ on the outer surface $R=B$, or by applying a uniformly distributed axial shear traction to the outer surface of the cylinder and assuming that the radial traction is zero there (cf. [10, p. 263]). The cylinder is assumed sufficiently long so that end effects are negligible.

For an isotropic incompressible or compressible elastic cylinder of arbitrary cross section, Knowles $[8,9]$ determined conditions on the strain-energy density of the material that ensure an anti-plane shear deformation, that is, a deformation with a single out-of-plane displacement that depends only on the in-plane coordinates, will be possible. The formulation in $[8,9]$ employs a rectangular Cartesian coordinate system. As discussed in [8,9], not all incompressible or compressible materials can sustain finite anti-plane shear deformations. The classes of materials that do give rise to such deformations are characterized in $[8,9]$. However, for a circular cylinder 
composed of an arbitrary incompressible isotropic elastic material, an exact solution can be obtained describing axisymmetric anti-plane shear deformations. (See [10, pp. 296-303; 3, Sec. 3.8] or Adkins [1]). These deformations, also termed telescopic shear $[11,12]$, are of the form $(2.1)$ with

$$
r=R .
$$

Thus the deformed configuration is again a hollow circular cylinder that undergoes no volume change. For a cylinder composed of a compressible material, there exists no global solution describing axisymmetric anti-plane shear deformations. In general, under axial shear conditions, the cylinder will undergo both an in-plane deformation $r(R)$ and an out-of-plane deformation with displacement field $w(R)$. These quantities are governed by two coupled nonlinear ordinary differential equations, one of which is second-order and the other first-order (see equations (2.26), (2.24) below). These equations also depend on the form of the strain-energy density function, $W$.

The strain-energy density per unit undeformed volume for isotropic elastic compressible materials is given by

$$
W=W\left(I_{1}, I_{2}, I_{3}\right),
$$

where $I_{1}, I_{2}, I_{3}$ are the principal invariants of the left Cauchy-Green deformation tensor $\mathbf{B}=\mathbf{F F}^{\mathrm{T}}$, where $\mathbf{F}$ is the deformation gradient tensor. We have

$$
\begin{aligned}
& I_{1}=\operatorname{tr} \mathbf{B}=\lambda_{1}^{2}+\lambda_{2}^{2}+\lambda_{3}^{2}, \\
& I_{2}=\operatorname{tr} \mathbf{B}^{*}=\lambda_{1}^{2} \lambda_{2}^{2}+\lambda_{2}^{2} \lambda_{3}^{2}+\lambda_{3}^{2} \lambda_{1}^{2}, \\
& I_{3}=\operatorname{det} \mathbf{B}=\lambda_{1}^{2} \lambda_{2}^{2} \lambda_{3}^{2},
\end{aligned}
$$

where $\mathbf{B}^{*}=(\operatorname{det} \mathbf{B}) \mathbf{B}^{-1}$ is the adjoint of $\mathbf{B}$ and $\lambda_{i}(i=1,2,3)$ are the principal stretches. The corresponding response equation for the Cauchy stress tensor $\mathbf{T}$ is (see e.g., [16])

$$
\mathbf{T}=\beta_{0} \mathbf{1}+\beta_{1} \mathbf{B}+\beta_{-1} \mathbf{B}^{-1},
$$

where 1 is the unit tensor. Here, the elastic response functions $\beta_{s}=\beta_{s}\left(I_{1}, I_{2}, I_{3}\right)$ $(s=0,1,-1)$ are given by

$$
\begin{gathered}
\beta_{0}=2 I_{3}^{-1 / 2}\left[I_{2} W_{2}+I_{3} W_{3}\right], \\
\beta_{1}=2 I_{3}^{-1 / 2} W_{1}, \quad \beta_{-1}=-2 I_{3}^{1 / 2} W_{2},
\end{gathered}
$$

where $W_{i}=\partial W / \partial I_{i} \quad(i=1,2,3)$. We shall assume that the strain-energy $W$ is sufficiently smooth and that $W$ and the stress $\mathbf{T}$ vanish in the undeformed state where $I_{1}=I_{2}=3, I_{3}=1$, and so

$$
W(3,3,1)=0, \quad W_{1}(3,3,1)+2 W_{2}(3,3,1)+W_{3}(3,3,1)=0 .
$$

Corresponding to the deformation field (2.1), we have

$$
\mathbf{F}=\left(\begin{array}{ccc}
\dot{r} & 0 & 0 \\
0 & r / R & 0 \\
\dot{w} & 0 & 1
\end{array}\right),
$$




$$
\begin{aligned}
\mathbf{B} & =\left(\begin{array}{ccc}
\dot{r}^{2} & 0 & \dot{r} \dot{w} \\
0 & r^{2} / R^{2} & 0 \\
\dot{r} \dot{w} & 0 & \dot{w}^{2}+1
\end{array}\right), \\
\mathbf{B}^{-1} & =\left(\begin{array}{ccc}
\left(\dot{w}^{2}+1\right) / \dot{r}^{2} & 0 & -\dot{w} / \dot{r} \\
0 & R^{2} / r^{2} & 0 \\
-\dot{w} / \dot{r} & 0 & 1
\end{array}\right) \\
I_{1} & =1+\frac{r^{2}}{R^{2}}+\dot{r}^{2}+\dot{w}^{2}, \\
I_{2} & =\frac{r^{2}}{R^{2}}+\dot{r}^{2}+\dot{r}^{2} \frac{r^{2}}{R^{2}}+\dot{w}^{2} \frac{r^{2}}{R^{2}} \\
I_{3} & =\dot{r}^{2} \frac{r^{2}}{R^{2}},
\end{aligned}
$$

where $\dot{w} \equiv d w / d R$. Substitution from (2.10), (2.11) into (2.6) yields the physical components of the Cauchy stress $\mathbf{T}$ as

$$
\begin{aligned}
& T_{r r}=\beta_{0}+\beta_{1} \dot{r}^{2}+\beta_{-1}\left(\dot{w}^{2}+1\right) / \dot{r}^{2}, \\
& T_{\theta \theta}=\beta_{0}+\beta_{1} \frac{r^{2}}{R^{2}}+\beta_{-1} \frac{R^{2}}{r^{2}}, \\
& T_{z z}=\beta_{0}+\beta_{1}\left(\dot{w}^{2}+1\right)+\beta_{-1}, \\
& T_{r z}=\beta_{1} \dot{r} \dot{w}-\beta_{-1} \frac{\dot{w}}{\dot{r}}, \\
& T_{r \theta}=0, \quad T_{\theta z}=0,
\end{aligned}
$$

where the $\beta_{s}(s=0,1,-1)$ given by (2.7) are evaluated at the values of the invariants given by $(2.12)-(2.14)$. Note that since $r=r(R)$, it is convenient to consider $\mathbf{T}=\mathbf{T}(R)$ rather than the more conventional $\mathbf{T}=\mathbf{T}(r)$.

The equilibrium equations in the absence of body forces are

$$
\operatorname{div} \mathbf{T}=\mathbf{0} \text {, }
$$

which, for this deformation, reduce to the following two equations:

$$
\begin{gathered}
\frac{\partial T_{r r}}{\partial r}+\frac{1}{r}\left(T_{r r}-T_{\theta \theta}\right)=0, \\
\frac{\partial T_{r z}}{\partial r}+\frac{T_{r z}}{r}=0 .
\end{gathered}
$$

We observe that equation (2.22) can be written in the form

$$
\frac{d}{d r}\left(r T_{r z}\right)=0
$$

so that on integrating and using (2.18), (2.14), (2.7), we arrive at the first-order nonlinear ordinary differential equation

$$
\dot{w}\left[R W_{1}+\frac{r^{2}}{R} W_{2}\right]=K,
$$


where $K$ is a constant and $W_{1}, W_{2}$ are evaluated at the values of the invariants given by (2.12)-(2.14).

Using the chain rule, we write $(2.21)$ as

$$
\frac{\partial T_{r r}}{\partial R}+\frac{\dot{r}}{r}\left(T_{r r}-T_{\theta \theta}\right)=0 .
$$

On substitution from (2.15), (2.16), (2.7) into (2.25), we obtain the second-order nonlinear ordinary differential equation

$$
\begin{aligned}
& \frac{d}{d R}\left[\frac{R \dot{r}}{r} W_{1}+\left(\frac{R \dot{r}}{r}+\frac{r \dot{r}}{R}\right) W_{2}+\frac{r \dot{r}}{R} W_{3}\right]+W_{1}\left(\frac{R \dot{r}^{2}}{r^{2}}-\frac{1}{R}\right)+W_{2}\left(\frac{R \dot{r}^{2}}{r^{2}}-\frac{1}{R}-\frac{\dot{w}^{2}}{R}\right) \\
& \quad=0,
\end{aligned}
$$

where again $W_{i}(i=1,2,3)$ are evaluated at the values $(2.12)-(2.14)$ for the invariants, and the ordinary derivative symbol is used since $r=r(R), w=w(R)$. Equations (2.24), (2.26) are a coupled pair of (highly) nonlinear ordinary differential equations for the unknown functions $r(R)$ and $w(R)$. These equations may also be obtained from an analysis presented by Ogden [10, pp. 263-264] in terms of principal stretches. The task of obtaining analytical solutions to the system (2.24), (2.26), even for simple forms of the strain-energy, is formidable. The recent paper [15] describes a numerical approach to solving an equivalent pair of equations for the case of a particular strain-energy density $W$.

To complete the formulation of the boundary-value problem, we assume the latter of the previously described boundary conditions at $R=B$. Therefore, in addition to $(2.2)$, we also require that

$$
\begin{array}{ll}
\text { (a) } T_{r r}(B)=0, & \text { (b) } T_{r z}(B)=T_{0}
\end{array}
$$

where $T_{0}>0$ is a given constant. The constant $K$ appearing in (2.24) can now be expressed in terms of $T_{0}$. Thus, on evaluating the left-hand side of (2.24) at $R=B$ and using $(2.18),(2.7),(2.27)(b)$ one finds that

$$
K=\frac{r(B) T_{0}}{2} \text {. }
$$

When $K$ and $T_{0}$ are related as in (2.28), the boundary condition (2.27)(b) is automatically satisfied. Thus the boundary-value problem is to solve the system (2.24), (2.26) for $w(R), r(R)$ on $A<R<B$ subject to the conditions (2.2), (2.27)(a).

3. Axisymmetric anti-plane shear: a necessary condition. We now proceed to determine a necessary condition on $W$ for axisymmetric anti-plane shear to be possible. On setting $r=R$ in (2.12)-(2.14), we find for axisymmetric anti-plane shear deformations that

$$
I_{1}=I_{2}=3+\dot{w}^{2}, \quad I_{3}=1,
$$

and so the deformation is isochoric. Also, on setting $r=R$ in (2.26) we obtain

$$
\frac{d}{d R}\left[W_{1}+2 W_{2}+W_{3}\right]-\frac{\dot{w}^{2}}{R} W_{2}=0
$$


On employing the chain rule, and noting that $\dot{w} \neq 0$ for a nontrivial solution, (3.2) can be written as

$$
2 \ddot{w}\left(\widehat{W}_{11}+2 \widehat{W}_{22}+3 \widehat{W}_{12}+\widehat{W}_{13}+\widehat{W}_{23}\right)-\frac{\dot{w}}{R} \widehat{W}_{2}=0 .
$$

Furthermore, on setting $r=R$ in (2.24) and using (2.28) with $r=R$, we find that

$$
\dot{w}\left(\widehat{W}_{1}+\widehat{W}_{2}\right)=\frac{B T_{0}}{2 R} \text {. }
$$

It will be assumed henceforth that $\dot{w} \neq$ constant and so $\ddot{w} \neq 0$. (Clearly $\dot{w}=$ constant would not be consistent with (3.4).) In (3.3), (3.4) we have introduced the notation $W_{i j}=\partial^{2} W / \partial I_{i} \partial I_{j}$, and the superposed hat is used to indicate that the corresponding quantities are evaluated at the values (3.1) for the invariants $I_{i} \quad(i=$ $1,2,3)$. The conditions (3.3), (3.4) are necessary conditions on $W$ for axisymmetric finite anti-plane shear to be possible. Condition (3.3) is a second-order nonlinear ordinary differential equation for $w(R)$ on $A<R<B$, and its solution must be compatible with that of the first-order nonlinear ordinary differential equation (3.4). The implications of (3.3), (3.4) are our concern in the remainder of this paper.

When $r=R$, the corresponding stresses follow from (2.15)-(2.19) as

$$
\begin{aligned}
T_{r r} & =\widehat{\beta}_{0}+\widehat{\beta}_{1}+\widehat{\beta}_{-1}\left(\dot{w}^{2}+1\right), \\
T_{\theta \theta} & =\widehat{\beta}_{0}+\widehat{\beta}_{1}+\widehat{\beta}_{-1}, \\
T_{z z} & =\widehat{\beta}_{0}+\widehat{\beta}_{1}\left(\dot{w}^{2}+1\right)+\widehat{\beta}_{-1}, \\
T_{r z} & =\dot{w}\left(\widehat{\beta}_{1}-\widehat{\beta}_{-1}\right), \\
T_{r \theta} & =0, \quad T_{\theta z}=0,
\end{aligned}
$$

where $\widehat{\beta}_{s}=\widehat{\beta}_{s}\left(3+\dot{w}^{2}, 3+\dot{w}^{2}, 1\right)$ are given by (2.7). For any $W$ for which (3.3), (3.4) hold on $A<R<B$, the boundary condition (2.27)(a) will be satisfied if

$$
\left.\left(\widehat{W}_{1}+2 \widehat{W}_{2}+\widehat{W}_{3}\right)\right|_{R=B}=0,
$$

where we recall that $B$ is the outer radius of the tube. Observe that when $r=R$, the first of the boundary conditions (2.2) at the inner radius $R=A$ is automatically satisfied and so the remaining boundary condition is

$$
w(A)=0 .
$$

Thus solutions $w(R)$ to (3.3), (3.4) on $A<R<B$ are sought, subject to the boundary conditions (3.10), (3.11).

In what follows, it will be convenient for us to consider the implications of differentiating both sides of (3.4) with respect to $R$. Performing this operation and using the chain rule, we obtain

$$
\left(\widehat{W}_{1}+\widehat{W}_{2}\right) \ddot{w}=-\frac{B T_{0}}{2 R^{2}}-2 \dot{w}^{2} \ddot{w}\left(\widehat{W}_{11}+2 \widehat{W}_{12}+\widehat{W}_{22}\right) .
$$

Furthermore, we will write (3.4) as

$$
\dot{w}=\frac{B T_{0}}{2 R\left(\widehat{W}_{1}+\widehat{W}_{2}\right)},
$$


provided that

$$
\widehat{W}_{1}+\widehat{W}_{2} \neq 0 \text { on } A<R<B \text {. }
$$

Since $W$ is assumed sufficiently smooth, it follows from (3.14) that $\widehat{W}_{1}+\widehat{W}_{2}$ does not change sign on $A<R<B$ and so, without loss of generality, we take

$$
\widehat{W}_{1}+\widehat{W}_{2}>0 \text { on } A<R<B \text {. }
$$

Note that (3.15) would also follow as a consequence of the Baker-Ericksen inequality.

To conclude this section, we write the stresses (3.5)-(3.9) in a simplified form. On substitution from (2.7) into (3.5)-(3.8), and then using (3.13) in (3.8), we find that

$$
\begin{aligned}
T_{r r} & =2\left[\widehat{W}_{1}+2 \widehat{W}_{2}+\widehat{W}_{3}\right], \\
T_{\theta \theta} & =2\left[\widehat{W}_{1}+\left(2+\dot{w}^{2}\right) \widehat{W}_{2}+\widehat{W}_{3}\right], \\
T_{z z} & =2\left[\left(1+\dot{w}^{2}\right) \widehat{W}_{1}+\left(2+\dot{w}^{2}\right) \widehat{W}_{2}+\widehat{W}_{3}\right], \\
T_{r z} & =B T_{0} / R, \quad T_{r \theta}=0, \quad T_{\theta z}=0 .
\end{aligned}
$$

4. A subclass of materials. We begin our analysis of (3.3), (3.4) by considering materials for which the dependence of $W$ on $I_{1}$ and $I_{2}$ is linear so that

$$
\widehat{W}_{11}=0, \quad \widehat{W}_{22}=0, \quad \widehat{W}_{12}=0 .
$$

On using (4.1) in (3.12) we obtain

$$
\ddot{w}=-\frac{B T_{0}}{2 R^{2}\left(\widehat{W}_{1}+\widehat{W}_{2}\right)},
$$

again provided that (3.15) holds. Employing (4.1), (3.13), and (4.2) in (3.3) we find that

$$
2 \widehat{W}_{13}+2 \widehat{W}_{23}+\widehat{W}_{2}=0
$$

Equation (4.3) is a nonlinear ordinary differential equation for $w(R)$ on $A<R<B$, and its solution must be compatible with that of (3.13) subject to (3.15).

Since $W$ is linear in $I_{1}$ and $I_{2}$, we may write $W$ as

$$
W=\frac{\mu}{2}\left[H_{1}\left(I_{3}\right)\left(I_{1}-3\right)+H_{2}\left(I_{3}\right)\left(I_{2}-3\right)+H_{3}\left(I_{3}\right)\right],
$$

where $\mu>0$ is constant. Thus

$$
\begin{gathered}
W_{1}=\frac{\mu}{2} H_{1}\left(I_{3}\right), \quad W_{2}=\frac{\mu}{2} H_{2}\left(I_{3}\right), \\
W_{3}=\frac{\mu}{2}\left[H_{1}^{\prime}\left(I_{3}\right)\left(I_{1}-3\right)+H_{2}^{\prime}\left(I_{3}\right)\left(I_{2}-3\right)+H_{3}^{\prime}\left(I_{3}\right)\right] .
\end{gathered}
$$

The prime notation denotes differentiation with respect to argument. To satisfy the normalization conditions (2.8), we see that the functions $H_{i}\left(I_{3}\right)(i=1,2,3)$ must be such that

$$
\text { (a) } H_{3}(1)=0, \quad \text { (b) } H_{1}(1)+2 H_{2}(1)+H_{3}^{\prime}(1)=0 \text {. }
$$

On substitution from (4.4), (4.5) into (4.3) and (3.15), and evaluating at the values (3.1), we find that

$$
2 H_{1}^{\prime}(1)+2 H_{2}^{\prime}(1)+H_{2}(1)=0
$$


and

$$
H_{1}(1)+H_{2}(1)>0
$$

Thus (4.7), (4.8) are necessary conditions on $H_{\alpha}\left(I_{3}\right)(\alpha=1,2)$ for axisymmetric anti-plane shear to be possible for the class of materials (4.4). Observe that we now have two algebraic conditions that must be satisfied instead of the differential equations (3.3), (3.4). An equivalent set of conditions obtained directly from the considerations of [9] can be found in [13].

Now (3.13) and the boundary condition (3.11) determine the out-of-plane displacement $w(R)$ for any $W$ of the form (4.4) as

$$
w(R)=\frac{B T_{0}}{\mu\left[H_{1}(1)+H_{2}(1)\right]} \ln \left(\frac{R}{A}\right) .
$$

By virtue of (4.8) we observe from (4.9) that, since $T_{0}>0$, we have $w(R)>0$ on $A<R<B$ as expected on physical grounds. Similar results have been obtained in $[11,12]$ for different sets of boundary conditions (see also [13]). It remains to satisfy the boundary condition (3.10) of zero radial traction on the outer surface. On using (3.1), (4.5), (4.6)(b), one finds that $(3.10)$ is satisfied if

$$
H_{1}^{\prime}(1)+H_{2}^{\prime}(1)=0 \text {. }
$$

On combining (4.7), (4.8), (4.10), we obtain

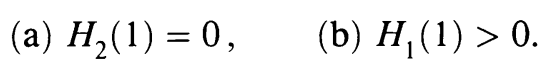

Thus, in summary, provided that (4.10), (4.11) hold, any $W$ of the form (4.4) will allow axisymmetric anti-plane shear deformations of the cylinder arising from a uniform shear traction applied to its lateral surface, with the radial traction vanishing there. The out-of-plane displacement $w(R)$ is then determined from (4.9), (4.11) as

$$
w(R)=\frac{B T_{0}}{\mu H_{1}(1)} \ln \left(\frac{R}{A}\right) .
$$

The corresponding stresses may then be found from (3.16)-(3.19). On using (4.10), (4.11), (4.12), we obtain

$$
\begin{gathered}
T_{r r}=0, \quad T_{\theta \theta}=0, \quad T_{r \theta}=0, \quad T_{\theta z}=0, \\
T_{z z}=\frac{B^{2} T_{0}^{2}}{\mu H_{1}(1) R^{2}}, \quad T_{r z}=\frac{B T_{0}}{R} .
\end{gathered}
$$

We observe that the axial stress $T_{z z}$, depending on the prescribed shear traction $T_{0}$ in a quadratic manner, represents a normal stress effect characteristic of finite elasticity theory. By virtue of $(4.11)$ (b) this axial stress is tensile.

As an example, we consider the Hadamard material [17]

$$
W=c_{1}\left(I_{1}-3\right)+c_{2}\left(I_{2}-3\right)+H\left(I_{3}\right),
$$

where $c_{1}, c_{2}$ are constants and $H\left(I_{3}\right)$ is, as yet, an arbitrary function. This is of the form (4.4) if we choose
(a) $H_{1}\left(I_{3}\right)=\frac{2 c_{1}}{\mu}$,
(b) $H_{2}\left(I_{3}\right)=\frac{2 c_{2}}{\mu}$,
(c) $H_{3}\left(I_{3}\right)=\frac{2 H\left(I_{3}\right)}{\mu}$. 
To ensure that the normalization conditions (4.6) hold, we require

$$
H(1)=0, \quad H^{\prime}(1)+c_{1}+2 c_{2}=0 .
$$

With $H_{i}\left(I_{3}\right)$ given by (4.16), it is seen that (4.10) is identically satisfied, and (4.11) holds provided

$$
c_{2}=0
$$

and

$$
c_{1}>0 \text {. }
$$

This is consistent with the result of Knowles in [9] who found that (4.18) is necessary and sufficient for an arbitrary shaped cylinder composed of a Hadamard material to sustain an arbitrary anti-plane shear deformation. The out-of-plane displacement for a Hadamard material satisfying (4.18), (4.19) is given by (4.12), (4.16)(a) as

$$
w(R)=\frac{B T_{0}}{2 c_{1}} \ln \left(\frac{R}{A}\right) .
$$

The nonzero stresses, given by (4.14), (4.16)(a) are

$$
T_{z z}=\frac{B^{2} T_{0}^{2}}{2 c_{1} R^{2}}, \quad T_{r z}=\frac{B T_{0}}{R} .
$$

Motivated by this example, we return to the material model (4.4) and observe from (4.11)(a) that, for materials for which $H_{2}\left(I_{3}\right)=c$ ( $c$ constant) to sustain axisymmetric finite anti-plane shear deformations, we must have $c \equiv 0$. If in addition to $H_{2}\left(I_{3}\right) \equiv 0, H_{1}\left(I_{3}\right)$ is constant $(>0)$, then (4.10), (4.11) are automatically satisfied, and so axisymmetric anti-plane shear is always possible for materials for which $W$ has the form

$$
W=\frac{\mu}{2}\left[k\left(I_{1}-3\right)+H\left(I_{3}\right)\right], \quad(k>0 \text { constant }),
$$

where

$$
H(1)=0, \quad H^{\prime}(1)+k=0,
$$

so that the normalization conditions (4.6) hold. Of course the materials described by (4.22), (4.23) are again Hadamard materials. A variety of constitutive laws of the form (4.22) have been proposed in the literature. These include the strain-energy functions proposed by Blatz [18],

$$
W=\frac{\mu}{2}\left[\left(I_{1}-3\right)-\frac{2}{1-2 \nu} \ln I_{3}^{1 / 2}+\frac{4 \nu}{1-2 \nu}\left(I_{3}^{1 / 2}-1\right)\right] ;
$$

by Blatz [19, p. 30],

$$
W=\frac{\mu}{2}\left(I_{1}-3 I_{3}^{1 / 3}\right)+\frac{\kappa}{m}\left(I_{3}^{1 / 2}-\frac{m}{m-1}+\frac{I_{3}^{1 / 2(1-m)}}{m-1}\right) ;
$$

by Ogden [20] (see also [10, p. 519; 21, p. 573]),

$$
W=\frac{\mu}{2}\left(I_{1}-3 I_{3}^{1 / 3}\right)+\frac{\kappa}{9}\left(\frac{I_{3}^{-9 / 2}}{9}+\ln I_{3}^{1 / 2}-\frac{1}{9}\right) ;
$$


and by Christensen [22],

$$
W=\frac{\mu}{2}\left(I_{1}-3\right)-\frac{3}{2} \kappa \ln I_{3}^{1 / 2}+\frac{9}{4}\left(\kappa-\frac{2}{3} \mu\right)\left(I_{3}^{1 / 3}-1\right),
$$

where, in all cases, the corresponding constant $k$ in (4.22) has value unity, and it is readily verified that $(4.23)$ is satisfied. On using $(4.10),(4.11)$, it can be readily verified that axisymmetric anti-plane shear also is always possible for the generalized Blatz-Ko material [23],

$$
\begin{aligned}
W= & \frac{\mu}{2} f\left\{I_{1}-1-\frac{1}{\nu}+\frac{(1-2 \nu)}{\nu} I_{3}^{-\nu /(1-2 \nu)}\right\} \\
& +\frac{\mu}{2}(1-f)\left\{\frac{I_{2}}{I_{3}}-1-\frac{1}{\nu}+\frac{(1-2 \nu)}{\nu} I_{3}^{\nu /(1-2 \nu)}\right\},
\end{aligned}
$$

and the polynomial material proposed by Levinson and Burgess [24],

$$
\begin{aligned}
W=\frac{\mu}{2}\left[f\left(I_{1}-3\right)+(1-f)\left(\frac{I_{2}}{I_{3}}-3\right)+\right. & 2(1-2 f)\left(I_{3}^{1 / 2}-1\right) \\
& \left.+\left(2 f+\frac{4 \nu-1}{1-2 \nu}\right)\left(I_{3}^{1 / 2}-1\right)^{2}\right],
\end{aligned}
$$

provided that the constant $f=1$, in which case $(4.28),(4.29)$ are also of the form (4.22) with $k=1$. The constants $\mu, \nu, \kappa=2 \mu(1+\nu) /(3(1-2 \nu))$ appearing in the strain-energy functions (4.24)-(4.29) are the shear modulus, Poisson's ratio, and bulk modulus for infinitesimal deformations respectively, so that $\mu>0,0<\nu<1 / 2$, $\kappa>0$. As described in [19], the constant $m>2 / 3 \quad(m \neq 1)$ appearing in (4.25) is a parameter arising from molecular considerations. A similar result for (4.28), (4.29) with $f=1$ was obtained previously in [11] for different boundary conditions (see also [13]). The out-of-plane displacement for the materials described by (4.24)-(4.29) is determined from (4.12) or (4.20) as

$$
w(R)=\frac{B T_{0}}{\mu} \ln \left(\frac{R}{A}\right),
$$

while the nonzero stresses (4.14) are

$$
T_{z z}=\frac{B^{2} T_{0}^{2}}{\mu R^{2}}, \quad T_{r z}=\frac{B T_{0}}{R} .
$$

Observe that when $H_{1}\left(I_{3}\right)$ in (4.4) is not constant (and $\left.H_{2}\left(I_{3}\right) \equiv 0\right)$, the conditions (4.10), (4.11) require that

$$
H_{1}(1)>0, \quad H_{1}^{\prime}(1)=0 .
$$

The normalization conditions (4.6) become

$$
H_{3}(1)=0, \quad H_{1}(1)+H_{3}^{\prime}(1)=0 \text {. }
$$

Thus (4.32), (4.33) are necessary in order for axisymmetric anti-plane shear to be possible for a material with

$$
W=\left(\frac{\mu}{2}\right)\left[H_{1}\left(I_{3}\right)\left(I_{1}-3\right)+H_{3}\left(I_{3}\right)\right]
$$


This result was obtained in a different way in [11] (see also [13]). The out-of-plane displacement is again given by (4.12), while the nonzero stresses are given by (4.14).

In the material models described by (4.22) and (4.34), the function $\mathrm{H}_{2}\left(I_{3}\right)$ of (4.4) is taken to be zero and so $W$ does not depend on $I_{2}$. Strain-energy densities $W$ which do depend on $I_{2}$, however, are also admissible. For example, if

$$
H_{1}\left(I_{3}\right)=k_{1}, \quad H_{2}\left(I_{3}\right) \neq \text { constant },
$$

then (4.10), (4.11) read

$$
H_{2}^{\prime}(1)=0, \quad H_{2}(1)=0, \quad k_{1}>0 .
$$

A simple choice for $H_{2}\left(I_{3}\right)$ satisfying (4.36) is

$$
H_{2}\left(I_{3}\right)=\beta\left(I_{3}-1\right)^{\alpha},
$$

where $\alpha>1, \beta \neq 0$ are arbitrary constants. Using (4.36), (4.35), we see from (4.6) that the remaining function $H_{3}\left(I_{3}\right)$ in (4.4) is then chosen so that the normalization conditions

$$
H_{3}(1)=0, \quad H_{3}^{\prime}(1)=-k_{1}
$$

are satisfied. The out-of-plane displacement is given by (4.9), which in view of (4.35), (4.36) reduces to

$$
w(R)=\frac{B T_{0}}{\mu k_{1}} \ln \left(\frac{R}{A}\right) .
$$

The nonzero stresses $(4.14)$ are

$$
T_{z z}=\frac{B^{2} T_{0}^{2}}{\mu k_{1} R^{2}}, \quad T_{r z}=\frac{B T_{0}}{R} .
$$

To conclude this section, we note that the function $\mathrm{H}_{3}\left(I_{3}\right)$ in (4.4) does not enter into the conditions (4.10), (4.11). However, it should be observed that a $W$ that depends on $I_{3}$ only is not admissible, since (4.4) would then yield $H_{1} \equiv 0, H_{2} \equiv 0$, which do not satisfy (4.11).

5. A more general class of materials. The task of seeking a representation for the most general form of $W$ for which (3.3), (3.4) are satisfied is formidable. Here we shall pursue a more modest objective, namely, that of broadening the subclass of materials discussed in Sec. 4.

Simplification of (3.3) to the more tractable (4.3), which contains no explicit dependence on derivatives of $w$, relied upon deducing the result (4.2) from (3.12). The assumption (4.1) that $W$ depended linearly on $I_{1}$ and $I_{2}$ made this possible. However, the result (4.2) can be obtained from (3.12) by merely requiring that

$$
\widehat{W}_{11}+2 \widehat{W}_{12}+\widehat{W}_{22}=0
$$

instead of the more restrictive conditions $\widehat{W}_{\alpha \beta}=0 \quad(\alpha, \beta=1,2)$. On employing (5.1), (4.2), (3.13) in (3.3) we find that

$$
2\left(\widehat{W}_{22}+\widehat{W}_{12}+\widehat{W}_{13}+\widehat{W}_{23}\right)+\widehat{W}_{2}=0 .
$$


Derived under the assumption that (5.1) holds, condition (5.2) is the analog of (4.3). The restriction (3.14) is still required to hold since this was used in deriving (3.13), (4.2). As pointed out in Sec. 3, we may, without loss of generality, replace (3.14) by (3.15). With this motivation, we thus consider the class of materials for which $W$ can be written as

$$
W=\left(\frac{\mu}{2}\right)\left[P\left(I_{1}-I_{2}, I_{3}\right)\left(I_{1}^{\cdot}-3\right)+Q\left(I_{1}-I_{2}, I_{3}\right)\left(I_{2}-3\right)+R\left(I_{1}-I_{2}, I_{3}\right)\right],
$$

where $\mu>0$, and $P, Q, R$ are sufficiently smooth functions of the arguments indicated in (5.3). It is readily verified that $W$ as given in (5.3) satisfies

$$
W_{11}+2 W_{12}+W_{22}=0 \text {, }
$$

so that, in particular, (5.1) holds for such $W$. The normalization conditions (2.8) will be satisfied by (5.3) if

$$
R(0,1)=0
$$

and

$$
P(0,1)+2 Q(0,1)-R_{1}(0,1)+R_{2}(0,1)=0,
$$

where, for any arbitrary function $F\left(I_{1}-I_{2}, I_{3}\right)$, the notation

$$
F_{1}(x, y)=\frac{\partial}{\partial x} F(x, y), \quad F_{2}(x, y)=\frac{\partial}{\partial y} F(x, y)
$$

is used to denote the derivative of $F\left(I_{1}-I_{2}, I_{3}\right)$ with respect to its arguments.

Our task now is to find conditions on the functions $P, Q, R$ appearing in (5.3) that will ensure (5.2) and (3.15) are satisfied. On substitution from (5.3) into (5.2), one finds that

$$
\begin{aligned}
& 2\left\{P_{2}(0,1)+Q_{2}(0,1)-\left[P_{1}(0,1)+Q_{1}(0,1)\right]\right\} \\
& \quad+Q(0,1)-R_{1}(0,1)-\left[P_{1}(0,1)+Q_{1}(0,1)\right] \dot{w}^{2}=0 .
\end{aligned}
$$

Since we have assumed that $\dot{w} \neq$ constant (see the remark following (3.4), (5.8) implies that

$$
P_{1}(0,1)+Q_{1}(0,1)=0 .
$$

Using (5.9), the condition (5.8) now reads

$$
2 P_{2}(0,1)+2 Q_{2}(0,1)+Q(0,1)-R_{1}(0,1)=0 .
$$

On substitution from (5.3) into (3.15), one also finds that

$$
P(0,1)+Q(0,1)>0 .
$$

Thus a cylindrical tube composed of a material described by a strain-energy function $W$ of the form (5.3), with $P, Q$ chosen so that (5.9) is satisfied, can sustain axisymmetric finite anti-plane shear deformations provided (5.10), (5.11) hold.

It remains to examine the possibility of satisfying the boundary conditions (3.10), (3.11) and to determine the out-of-plane displacement $w(R)$. From (3.13), (3.11), we obtain

$$
w(R)=\frac{B T_{0}}{\mu[P(0,1)+Q(0,1)]} \ln \left(\frac{R}{A}\right)
$$


for any $W$ of the form (5.3). By virtue of (5.11), we see that $w(R)>0$ on $A<R<$ $B$. On substitution from (5.3) into the boundary condition of zero radial traction (3.10), and using (5.6), (5.9), one finds that

$$
P_{2}(0,1)+Q_{2}(0,1)=0 .
$$

Combining (5.13) and (5.10), we obtain

$$
Q(0,1)-R_{1}(0,1)=0 .
$$

Thus, in summary, provided that (5.9), (5.11), (5.13), and (5.14) hold, any $W$ of the form (5.3) will allow axisymmetric anti-plane shear deformations of the tube arising from a uniform shear traction applied to its outer lateral surface, with the radial traction vanishing there.

On substitution from (5.3) into (3.16)-(3.19), and using (5.6), (5.9), (5.11)-(5.14) we find that the only nonzero stresses are

$$
T_{z z}=\frac{B^{2} T_{0}^{2}}{\mu[P(0,1)+Q(0,1)] R^{2}}, \quad T_{r z}=\frac{B T_{0}}{R} .
$$

We note that if $P, Q, R$ in (5.3) are functions of $I_{3}$ only, we recover (4.4). In this case, $P_{1}=Q_{1}=R_{1} \equiv 0$, and all equations in this section are seen to reduce accordingly to the corresponding equations in Sec. 4 .

We shall not attempt to describe the most general class of functions $P, Q, R$ for which (5.9), (5.11), (5.13), and (5.14) are satisfied but rather indicate some of the possibilities. It will be seen that a wide variety of admissible strain-energies are included in the form (5.3). Suppose, for example, that we seek $P, Q$ in the separable form

$$
\begin{aligned}
& P\left(I_{1}-I_{2}, I_{3}\right)=F\left(I_{1}-I_{2}\right) U\left(I_{3}\right), \\
& Q\left(I_{1}-I_{2}, I_{3}\right)=G\left(I_{1}-I_{2}\right) V\left(I_{3}\right) .
\end{aligned}
$$

Then, on substitution into (5.9), (5.13), (5.11) we find that $F, G, U, V$ must satisfy

$$
\begin{gathered}
F^{\prime}(0) U(1)+G^{\prime}(0) V(1)=0, \\
F(0) U^{\prime}(1)+G(0) V^{\prime}(1)=0, \\
F(0) U(1)+G(0) V(1)>0 .
\end{gathered}
$$

To reduce (5.18)-(5.20) to restrictions on $U, V, U^{\prime}, V^{\prime}$ only, we choose

$$
\begin{aligned}
& F\left(I_{1}-I_{2}\right)=e^{\alpha\left(I_{1}-I_{2}\right)}, \\
& G\left(I_{1}-I_{2}\right)=e^{-\alpha\left(I_{1}-I_{2}\right)},
\end{aligned}
$$

where $\alpha \neq 0$ is an arbitrary constant. With this choice for $F$ and $G$, we find from $(5.18)-(5.20)$ that

$$
\begin{gathered}
U(1)-V(1)=0, \\
U^{\prime}(1)+V^{\prime}(1)=0, \\
U(1)+V(1)>0 .
\end{gathered}
$$


The choice

$$
\begin{aligned}
& U\left(I_{3}\right)=\gamma e^{\beta\left(I_{3}-1\right)}, \\
& V\left(I_{3}\right)=\gamma e^{-\beta\left(I_{3}-1\right)},
\end{aligned}
$$

where $\beta \neq 0, \gamma>0$ are arbitrary constants, is seen to satisfy (5.23)-(5.25). Thus the functional forms for $P, Q$ in (5.16), (5.17) are determined to be

$$
\begin{aligned}
& P\left(I_{1}-I_{2}, I_{3}\right)=\gamma e^{\alpha\left(I_{1}-I_{2}\right)} e^{\beta\left(I_{3}-1\right)}, \\
& Q\left(I_{1}-I_{2}, I_{3}\right)=\gamma e^{-\alpha\left(I_{1}-I_{2}\right)} e^{-\beta\left(I_{3}-1\right)} .
\end{aligned}
$$

It remains to find $R\left(I_{1}-I_{2}, I_{3}\right)$ satisfying (5.14) and the normalization conditions (5.5), (5.6). Using (5.28), (5.29), we find that $R$ must satisfy

$$
R_{1}(0,1)=\gamma, \quad R(0,1)=0, \quad R_{2}(0,1)=-2 \gamma,
$$

where we recall from (5.7) the definition of the subscript notation on $R\left(I_{1}-I_{2}, I_{3}\right)$. A choice for $R$ satisfying (5.30) is

$$
R\left(I_{1}-I_{2}, I_{3}\right)=\gamma\left[e^{\left(I_{1}-I_{2}\right)} e^{-2\left(I_{3}-1\right)}-1\right] .
$$

Combining (5.28), (5.29), (5.31) in (5.3) we conclude that a cylindrical tube composed of a material described by a strain-energy density $W$ of the form

$$
W=\frac{\mu}{2} \gamma\left[e^{\alpha\left(I_{1}-I_{2}\right)} e^{\beta\left(I_{3}-1\right)}\left(I_{1}-3\right)+e^{-\alpha\left(I_{1}-I_{2}\right)} e^{-\beta\left(I_{3}-1\right)}\left(I_{2}-3\right)+e^{\left(I_{1}-I_{2}\right)} e^{-2\left(I_{3}-1\right)}-1\right],
$$

with $\alpha \neq 0, \beta \neq 0, \gamma>0$ arbitrary constants, can sustain axisymmetric finite anti-plane shear deformations. Strain-energies with exponential terms have been successfully used in biomechanical modeling (see e.g., $[25,26]$ and the references cited therein). The out-of-plane displacement $w(R)$ for the material described by (5.32) follows from (5.12) as

$$
w(R)=\frac{B T_{0}}{2 \mu \gamma} \ln \left(\frac{R}{A}\right),
$$

while the nonzero stresses given by (5.15) are

$$
T_{z z}=\frac{B^{2} T_{0}^{2}}{2 \mu \gamma R^{2}}, \quad T_{r z}=\frac{B T_{0}}{R} .
$$

Returning to (5.18)-(5.20), we examine another possibility where $F, G$ are chosen as polynomials rather than as exponentials. For example, suppose that

$$
F\left(I_{1}-I_{2}\right)=c\left(I_{1}-I_{2}\right)^{r},
$$

where $c, r$ are arbitrary constants with $c \neq 0, r>1$, so that $F(0)=0, F^{\prime}(0)=0$. Then $(5.18)-(5.20)$ yield

$$
\begin{gathered}
G^{\prime}(0)=0, \quad V^{\prime}(1)=0, \\
G(0) V(1)>0 .
\end{gathered}
$$


Simple choices for $G, V$ satisfying (5.36), (5.37) are

$$
\begin{gathered}
G\left(I_{1}-I_{2}\right)=e^{p\left(I_{1}-I_{2}\right)}-p\left(I_{1}-I_{2}\right), \\
V\left(I_{3}\right)=e^{q\left(I_{3}-1\right)}-q\left(I_{3}-1\right),
\end{gathered}
$$

where $p, q$ are arbitrary nonzero constants. An alternative choice not involving exponentials is

$$
\begin{gathered}
G\left(I_{1}-I_{2}\right)=c_{1}\left(I_{1}-I_{2}\right)^{s}+k_{1}, \\
V\left(I_{3}\right)=c_{2}\left(I_{3}-1\right)^{t}+k_{2},
\end{gathered}
$$

where $c_{1}, c_{2}$ are arbitrary nonzero constants and $k_{1}, k_{2}, s>1, t>1$ are arbitrary constants with $k_{1} k_{2}>0$. Thus from (5.40), (5.41), (5.16), (5.17) we obtain

$$
\begin{gathered}
P\left(I_{1}-I_{2}, I_{3}\right)=c\left(I_{1}-I_{2}\right)^{r} U\left(I_{3}\right), \\
Q\left(I_{1}-I_{2}, I_{3}\right)=\left[c_{1}\left(I_{1}-I_{2}\right)^{s}+k_{1}\right]\left[c_{2}\left(I_{3}-1\right)^{t}+k_{2}\right],
\end{gathered}
$$

where $U\left(I_{3}\right)$ is still an arbitrary function.

Again the remaining function $R\left(I_{1}-I_{2}, I_{3}\right)$ is to be determined from (5.14) and the normalization conditions (5.5), (5.6). Using (5.42), (5.43) one finds that $R$ must satisfy

$$
R_{1}(0,1)=k_{1} k_{2}, \quad R(0,1)=0, \quad R_{2}(0,1)=-k_{1} k_{2} .
$$

A choice for $R\left(I_{1}-I_{2}, I_{3}\right)$ in terms of exponentials is again easily determined. Alternatively, it is readily verified that

$$
R\left(I_{1}-I_{2}, I_{3}\right)=k_{1} k_{2}\left(I_{1}-I_{2}\right) I_{3}-k_{1} k_{2}\left(I_{3}-1\right)
$$

satisfies (5.44). On combining (5.42), (5.43), (5.45) in (5.3) we conclude that an admissible choice for $W$ is

$$
\begin{aligned}
W=\frac{\mu}{2}\left\{c\left(I_{1}-I_{2}\right)^{r} U\left(I_{3}\right)\left(I_{1}-3\right)+\left[c_{1}\left(I_{1}-I_{2}\right)^{s}+k_{1}\right]\left[c_{2}\left(I_{3}-1\right)^{t}+k_{2}\right]\left(I_{2}-3\right)\right. \\
\left.+k_{1} k_{2}\left(I_{1}-I_{2}\right) I_{3}-k_{1} k_{2}\left(I_{3}-1\right)\right\},
\end{aligned}
$$

where $c, c_{1}, c_{2}$ are arbitrary nonzero constants, $k_{1}, k_{2}, r>1, s>1, t>1$ are arbitrary constants with $k_{1} k_{2}>0$, and $U\left(I_{3}\right)$ is an arbitrary function. The out-of-plane displacement follows from (5.12), (5.42), (5.43) as

$$
w(R)=\frac{B T_{0}}{\mu k_{1} k_{2}} \ln \left(\frac{R}{A}\right),
$$

while the nonzero stresses given by (5.15) are

$$
T_{z z}=\frac{B^{2} T_{0}^{2}}{\mu k_{1} k_{2} R^{2}}, \quad T_{r z}=\frac{B T_{0}}{R} .
$$

A similar argument in which the roles of $F, G$ and $U, V$ are interchanged in the preceding leads to another $W$ of the form (5.3) with

$$
\begin{gathered}
P\left(I_{1}-I_{2}, I_{3}\right)=\left[c_{1}\left(I_{1}-I_{2}\right)^{s}+k_{1}\right]\left[c_{2}\left(I_{3}-1\right)^{t}+k_{2}\right], \\
Q\left(I_{1}-I_{2}, I_{3}\right)=c\left(I_{1}-I_{2}\right)^{r} V\left(I_{3}\right),
\end{gathered}
$$


where $c, c_{1}, c_{2}$ are arbitrary nonzero constants, $k_{1}, k_{2}, r>1, s>1, t>1$ are arbitrary constants with $k_{1} k_{2}>0$, and $V\left(I_{3}\right)$ is arbitrary. In this case, one finds from (5.14), (5.5), (5.6) that the remaining function $R\left(I_{1}-I_{2}, I_{3}\right)$ must satisfy

$$
R_{1}(0,1)=0, \quad R(0,1)=0, \quad R_{2}(0,1)=-k_{1} k_{2} .
$$

A choice for $R$ satisfying $(5.51)$ is

$$
R\left(I_{1}-I_{2}, I_{3}\right)=\left(I_{1}-I_{2}\right)^{\alpha} H\left(I_{3}\right)+\gamma\left(I_{3}-1\right)^{\beta}-k_{1} k_{2}\left(I_{3}-1\right),
$$

where $\alpha>1, \beta>1$ are arbitrary constants, $\gamma$ is an arbitrary nonzero constant, and $H\left(I_{3}\right)$ is an arbitrary sufficiently smooth function. The explicit form for $W$ is then found on substitution from (5.49), (5.50), (5.52) into (5.3). The out-of-plane displacement is still given by (5.47) and the nonzero stresses by (5.48). Several other admissible choices for $W$ would follow on using (5.35), (5.39) instead of (5.40), (5.41) for $G, V$ respectively and by permutation among the various possibilities described above.

There are also admissible strain-energies of the form (5.3) where $P, Q$ are not separable, as was assumed in (5.16), (5.17). For example, it is readily verified that

$$
\begin{aligned}
& P\left(I_{1}-I_{2}, I_{3}\right)=\gamma e^{\alpha\left(I_{3}-1\right)\left(I_{1}-I_{2}\right)}, \\
& Q\left(I_{1}-I_{2}, I_{3}\right)=\delta e^{\beta\left(I_{3}-1\right)\left(I_{1}-I_{2}\right)},
\end{aligned}
$$

where $\alpha, \beta, \gamma, \delta$ are arbitrary nonzero constants, with $\gamma+\delta>0$, satisfy (5.9), (5.11), (5.13). The remaining function $R\left(I_{1}-I_{2}, I_{3}\right)$ is again determined from (5.14), (5.5), (5.6). Using (5.53), (5.54) one finds that $R$ must satisfy

$$
R_{1}(0,1)=\delta, \quad R(0,1)=0, \quad R_{2}(0,1)=-(\gamma+\delta) .
$$

A simple choice for $R$ satisfying (5.55) is, for example,

$$
R\left(I_{1}-I_{2}, I_{3}\right)=\delta I_{3}\left(I_{1}-I_{2}\right)-I_{3}^{\gamma+\delta} \text {. }
$$

The explicit form for $W$ is again found on substitution from (5.53), (5.54), (5.56) into (5.3). The out-of-plane displacement follows from (5.12), (5.53), (5.54),

$$
w(R)=\frac{B T_{0}}{\mu(\gamma+\delta)} \ln \left(\frac{R}{A}\right),
$$

while the nonzero stresses given by $(5.15),(5.53),(5.54)$ are

$$
T_{z z}=\frac{B^{2} T_{0}^{2}}{\mu(\gamma+\delta) R^{2}}, \quad T_{r z}=\frac{B T_{0}}{R} .
$$

We note that strain-energy density functions $W$ with exponential terms such as (5.53), (5.54) have also been used in biomechanical modeling [27].

Thus we have seen in Sec. 5 that the form (5.3) encompasses a wide variety of strain-energy density functions $W$ for which an axisymmetric finite anti-plane shear deformation of the tube is possible.

In conclusion, it is worthwhile observing that all the out-of-plane displacements $w(R)$ obtained explicitly in Secs. 4, 5 are logarithmic functions of $R$ and thus satisfy the axisymmetric form of Laplace's equation. Thus these displacements are identical to those of the corresponding linearized theory. The nonlinearity of the problem is reflected by the presence of the axial normal stress $T_{z z}$. 
Acknowledgment. This research was supported in part by the National Science Foundation under Grant MSM-89-04719 and by the U. S. Air Force Office of Scientific Research under Grant AFOSR-89-0470. The research of D. A. Polignone was also supported by a National Defense Science and Engineering Graduate Fellowship (NDSEGF) awarded by the U. S. Air Force.

Note added in proof. Since this paper was written, the authors have received a preprint [28] which describes classes of homogeneous, isotropic, compressible hyperelastic materials capable of sustaining states of finite anti-plane shear in cylindrical domains of arbitrary cross section. When the domain geometry is specialized to being a hollow circular cylinder and the deformations are restricted to be axisymmetric, some of the results established in Sec. 4 here can also be deduced from [28].

\section{REFERENCES}

[1] J. E. Adkins, Some generalizations of the shear problem for isotropic incompressible materials, Proc. Cambridge Philos. Soc. 50, 334-345 (1954)

[2] A. E. Green and J. E. Adkins, Large Elastic Deformations, Clarendon Press, Oxford, 1960

[3] A. E. Green and W. Zerna, Theoretical Elasticity, Oxford Univ. Press, London, 1968

[4] J. K. Knowles, The finite anti-plane shear field near the tip of a crack for a class of incompressible elastic solids, Internat. J. Fracture 13, 611-639 (1977)

[5] R. Abeyaratne and C. O. Horgan, Bounds on stress concentration factors in finite anti-plane shear, J. Elasticity 13, 49-61 (1983)

[6] A. H. Jafari, C. O. Horgan, and R. Abeyaratne, Finite anti-plane shear of an infinite slab with a traction-free elliptical cavity: bounds on the stress concentration factor, Internat. J. Non-Linear Mech. 19, 431-443 (1984)

[7] C. O. Horgan and S. A. Silling, Stress concentration factors in finite anti-plane shear: numerical calculations and analytical estimates, J. Elasticity 18, 83-91 (1987)

[8] J. K. Knowles, On finite anti-plane shear for incompressible elastic materials, J. Austral. Math. Soc. Ser. B 19, 400-415 (1976)

[9] J. K. Knowles, A note on anti-plane shear for compressible materials in finite elastostatics, J. Austral. Math. Soc. Ser. B 20, 1-7 (1977)

[10] R. W. Ogden, Non-linear Elastic Deformations, Ellis Horwood, Chichester, 1984

[11] A. Mioduchowski and J. B. Haddow, Finite telescopic shear of a compressible hyperelastic tube, Internat. J. Non-Linear Mech. 9, 209-220 (1974)

[12] M. G. Faulkner, Compressibility effects for nearly incompressible elastic solids, Appl. Sci. Res. 25, 328-336 (1972)

[13] V. K. Agarwal, On finite anti-plane shear for compressible elastic circular tube, J. Elasticity 9, 311-319 (1979)

[14] D. A. Polignone and C. O. Horgan, Pure torsion of compressible nonlinearly elastic circular cylinders, Quart. Appl. Math 49, 591-607 (1991)

[15] A. Ertepinar and G. Erarslanoglu, Finite anti-plane shear of compressible hyperelastic tubes, Internat. J. Engrg. Sci. 28, 399-406 (1990)

[16] C. Truesdell and W. Noll, The non-linear field theories of mechanics, Handbuch der Physik, III/3 (S. Flügge, ed.), Springer-Verlag, Berlin, 1965

[17] F. John, Plane elastic waves of finite amplitude: Hadamard materials and harmonic materials, Comm. Pure Appl. Math. 19, 309-341 (1966)

[18] P. J. Blatz, Application of finite elastic theory in predicting the performance of solid propeliane rocket motors, Calif. Inst. of Tech., GALCIT SM60-25, 1960

[19] P. J. Blatz, Application of large deformation theory to the thermo-mechanical behavior of rubberlike polymers-porous, unfilled, and filled, Rheology, Theory and Applications, vol. 5 (F. R. Eirich, ed.), Academic Press, New York, 1969, pp. 1-55

[20] R. W. Ogden, Large deformation isotropic elasticity: on the correlation of theory and experiment for compressible rubberlike solids, Proc. Roy. Soc. London Ser. A 328, 567-583 (1972) 
[21] J. B. Haddow and R. W. Ogden, Compression of bonded elastic bodies, J. Mech. Phys. Solids 36, 551-579 (1988)

[22] R. M. Christensen, $A$ two material constant, nonlinear elastic stress constitutive equation including the effect of compressibility, Mech. of Materials 7, 155-162 (1988)

[23] P. J. Blatz and W. L. Ko, Application of finite elasticity to the deformation of rubbery materials, Transactions of the Society of Rheology 6, 223-251 (1962)

[24] M. Levinson and I. W. Burgess, A comparison of some simple constitutive relations for slightly compressible rubber-like materials, Internat. J. Mech. Sci. 13, 563-572 (1971)

[25] Y. C. Fung, Biomechanics. Mechanical properties of living tissues, Springer-Verlag, Berlin, 1981

[26] M. F. Beatty, Topics in finite elasticity: hyperelasticity of rubber, elastomers, and biological tissueswith examples, Appl. Mech. Reviews 40, 1699-1734 (1987)

[27] P. F. Chu, Strain energy function for biological tissues, J. Biomechanics 3, 547-550 (1970)

[28] Q. Jiang and J. K. Knowles, A class of compressible elastic materials capable of sustaining finite anti-plane shear, J. Elasticity (in press) 